\title{
MicroRNA let-7b-regulated epidermal stem cell proliferation in hypertrophied anal papillae
}

\author{
HONG LU ${ }^{1 *}$, XIANG HE $^{1 *}$, QINGMING WANG ${ }^{1}$, DE ZHENG $^{1}$, YE HAN $^{1}$, WEI YANG $^{1}$ and TE LIU $^{2}$ \\ ${ }^{1}$ Department of Anorectal Dermatology, Shuguang Hospital, Shanghai University of Traditional Chinese Medicine, \\ Shanghai 201203; ${ }^{2}$ Shanghai Geriatric Institute of Chinese Medicine, Longhua Hospital, \\ Shanghai University of Traditional Chinese Medicine, Shanghai 200031, P.R. China
}

Received July 19, 2014; Accepted April 10, 2015

DOI: $10.3892 / \mathrm{mmr} .2015 .4017$

\begin{abstract}
The present study investigated the role of epidermal stem cell-expressed microRNA let-7 $b$ in the pathogenesis of hypertrophied anal papillae. Hypertrophied anal papillae were examined for the presence of epidermal stem cells. Epidermal stem cells were identified using flow cytometry and immunofluorescent staining for the cell surface markers, integrin $\alpha 6$ and integrin $\beta 1$ subunits. Expression levels of microRNA let- $7 b$ in $\alpha 6+/ \beta 1+$ and $\alpha 6-/ \beta 1$-cells were compared using reverse transcription-quantitative polymerase chain reaction and northern blotting. Lentivirus-mediated expression of microRNA let-7b in epidermal stem cells was utilized in order to study the effects of this microRNA on the cell cycle proteins, cyclin D1 (CCND1) and cyclin-dependent kinase 4 (CDK4). MicroRNA let-7b-overexpressing cells were examined using flow cytometry, in order to determine the effects of the microRNA on cell cycle progression. $\alpha 6+/ \beta 1+$ epidermal stem cells were identified in hypertrophic anal papillae. Following isolation and enrichment of the $\alpha 6+/ \beta 1+$ population, these cells were found to have a rapid rate of proliferation in vitro. The expression of cell cycle-related proteins was elevated in this population, compared with that in $\alpha 6-/ \beta 1$-cells. The expression of microRNA let- $7 b$ in $\alpha 6+/ \beta 1+$ epidermal stem cells was significantly lower than that in $\alpha 6-/ \beta 1$-cells. Two microRNA let-7b target genes, CCND1 and CDK4, were found to be
\end{abstract}

Correspondence to: Professor Te Liu, Shanghai Geriatric Institute of Chinese Medicine, Longhua Hospital, Shanghai University of Traditional Chinese Medicine, 365 Xiangyang Road, Shanghai 200031, P.R. China

E-mail: teliu79@126.com

Professor Wei Yang, Department of Anorectal Dermatology, Shuguang Hospital, Shanghai University of Traditional Chinese Medicine, 528 Zhangheng Road, Shanghai 201203, P.R. China

E-mail: weiyang@126.com

*Contributed equally

Key words: hypertrophied anal papillae, integrin $\alpha 6 / \beta 1$, epidermal stem cells, microRNA let-7b, proliferation upregulated in $\alpha 6+/ \beta 1+$ cells. When the exogenous precursor, microRNA let-7, was overexpressed in $\alpha 6+/ \beta 1+$ epidermal stem cells, the cell proliferation rate was significantly lower than that in cells expressing microRNA let-7 containing a mutated seed sequence. The addition of exogenous microRNA let-7 resulted in an increased expression level of mature microRNA let-7b, while the expression of CCND1 and CDK4 was reduced. Epidermal stem cells transfected with microRNA let- $7 b$ were arrested in the G2/M phase and the percentage of cells in S-phase was significantly reduced. In conclusion, let-7b expression results in upregulation of the cell cyclerelated proteins, CCND1 and CDK4, resulting in the excessive proliferation that leads to the formation of hypertrophic anal papillae.

\section{Introduction}

Anal papillomas are common, benign anal cysts. Previous studies have indicated that hypertrophied anal papillae arise as a result of proliferative inflammatory disease (1-3). Hypertrophied anal papillae are, in essence, skin tags that project up from the dentate line, or from the junction between the skin and the epithelial lining of the anus. They are often found as part of the classic triad of a chronic fissure; namely the fissure itself, with hypertrophied papilla above and a skin tag below (1-3). However, the mechanisms underlying the development of human hypertrophied anal papillae are poorly understood.

Epidermal stem cells (EpSCs) are skin tissue-specific adult stem cells with a strong proliferative capacity, which undergo asymmetric division (4-7). Following induction, EpSCs are able to differentiate into a variety of epidermal lineages in order to promote self-renewal and regeneration of the epidermis, and to promote wound healing $(5,6,8,9)$. Proliferation and differentiation of EpSCs may be regulated by a number of factors, including the stem cell microenvironment, or cellular factors and cell surface receptors $(5,6)$. Previous studies have found that the integrin family of proteins has a significant effect on EpSC proliferation and differentiation. Integrins have been utilized for the isolation and enrichment of EpSCs from tissues in vitro $(5,6,8-10)$. Integrins are cell surface receptors composed of $\alpha$ and $\beta$ subunits. To date, 18 different $\alpha$ subunits and $8 \beta$ subunits have been identified that may be combined to 
form a total of 24 different integrin receptors in mammals (9). The N-terminal region of the $\alpha$ subunit forms a domain that binds divalent cations and contains a highly conserved sequence, 'KXGFFKR', which is proximal to the cytoplasmic membrane and is involved in regulation of integrin activity (9). $\beta 1$ integrins form the largest subgroup of integrins. The 12 members of this group bind a variety of ligands. $\alpha 1 \beta 1, \alpha 2 \beta 1$, $\alpha 10 \beta 1$ and $\alpha 11 \beta 1$ primarily interact with collagen, an interaction that is conducive to cell proliferation. $\alpha 1 \beta 1, \alpha 2 \beta 1, \alpha 3 \beta 1$, $\alpha 6 \beta 1$ and $\alpha 7 \beta 1$ interact primarily with laminins, which are involved in adhesion to the basement membrane. $\alpha 4 \beta 1, \alpha 5 \beta 1$, $\alpha 8 \beta 1$ and $\alpha v \beta 1$ bind fibronectin, and $\alpha 9 \beta 1$ binds tenascins (9). In the intact epidermis, integrin expression occurs in the basal layer and outer root sheath of hair follicles. $\beta 1$ expression is predominantly confined to regions of the hair follicle bulge and epidermal prominences, while $\alpha 6$ integrin expression occurs in the outer root sheath of hair follicles and the outermost basal layer of the interfollicular epithelium, which is composed of hemidesmosomes. Integrins $\alpha 6$ and $\beta 1$ are used as molecular biomarkers of EpSCs $(9,10)$.

MicroRNAs (miRNAs) are a recently discovered class of naturally occurring, single-stranded, 21-23 nucleotide, non-coding RNAs $(11,12)$, which exist in a wide range of eukaryotic organisms (11-16). Each mammalian miRNA may prevent the translation of a number of downstream target mRNAs, which ultimately results in the inhibition of target gene expression (17-20). let-7 is a well-studied miRNA, known to be involved in cell cycle regulation and development, which is underexpressed in various cancers (21). Restoration of let-7 expression has been found to inhibit cancer growth by targeting various oncogenes and inhibiting key regulators of numerous mitogenic pathways (21-24). Yu et al (24) found that let-7 suppressed self-renewal and tumorigenicity of breast cancer cells by reducing H-RAS and high-mobility group AT-hook 2 (HMGA2) expression. Furthermore, Schultz et al (22) reported that let-7b, a member of the let-7 microRNA family, interfered with the proliferation and growth of primary malignant melanoma cells by targeting and suppressing important cell cycle molecules, such as cyclin D1 (CCND1). In addition, Dangi-Garimella et al (23) revealed that elevated let-7 expression inhibited HMGA2 expression and suppressed metastasis in breast cancer cells.

The present study aimed to establish the role of miRNA let $-7 b$ in regulation of integrin $\alpha 6+/ \beta 1+\mathrm{EpSC}$ proliferation and define its role in the formation of human hypertrophied anal papillae.

\section{Materials and methods}

Patients and ethics. Hypertrophic anal papilla tissue samples were obtained during surgery from five patients who had been diagnosed with mixed hemorrhoids or anal fistula. Two patients (1 male and 1 female) had archosyrinx, and three patients ( 2 males and 1 female) had mixed hemorrhoids. The median age of this population was 39 years old. All human materials were obtained from the Department of Anorectal Dermatology (Shuguang Hospital, Shanghai University of Traditional Chinese Medicine, Shanghai, China). All of the patients in the present study provided written informed consent. The study was approved by the ethics committee of Shanghai Geriatric
Institute of Chinese Medicine, Longhua Hospital, Shanghai University of Traditional Chinese Medicine (Shanghai, China).

Isolation of integrin $\alpha 6$ and integrin $\beta 1$ phenotype cells by a magnetic activated cell sorting system. Integrin $\alpha 6$ and Integrin $\beta 1$ subpopulation cells were isolated from primary cells from hypertrophic anal papilla tissues, using $4 \mu \mathrm{l}$ primary monoclonal antibody (rabbit anti-human Integrin $\alpha 6$-FITC, rabbit anti-human Integrin $\beta 1$-PE, eBioscience, Inc., San Diego, CA, USA), stored at $4^{\circ} \mathrm{C}$ in phosphate-buffered saline (PBS) for $30 \mathrm{~min}$ in a volume of $1 \mathrm{ml}$, as previously described $(4,25)$. Following this reaction, the cells were washed twice in PBS, and goat anti-rabbit secondary monoclonal antibodies conjugated to magnetic microbeads (Miltenyi Biotec, Auburn, CA, USA) were added, incubated at $10^{\circ} \mathrm{C}$ in PBS for $15 \mathrm{~min}$ and then washed twice in PBS. Single cells were plated at 1,000 cells/ml in Dulbecco's modified Eagle's medium (DMEM:F12; HyClone Laboratories, Inc., Logan, UT, USA), supplemented with $10 \%$ fetal bovine serum (FBS), $10 \mathrm{ng} / \mathrm{ml}$ basic fibroblast growth factor (bFGF), $10 \mathrm{ng} / \mathrm{ml}$ epidermal growth factor (EGF), $5 \mu \mathrm{g} / \mathrm{ml}$ insulin and $0.5 \%$ bovine serum albumin (BSA; all Sigma-Aldrich, St. Louis, MO, USA). All cells had been cultured under the same conditions until passage three, prior to subsequent experiments.

Recombinant lentivirus second generation vector construction. All steps of recombinant lentivirus packaging were conducted as previously described $(17,20)$. An RNAi pLL3.7 (LentiLox 3.7) letroviral system was used to create lentiviral virus vectors (GenePharma Co., Ltd., Shanghai, China). For vector pLL3.7-miR-let-7 (pre-microRNA let-7), an oligonucleotide pair for pre-miRNA of microRNA let-7, and linker sequences with HpaI and XhoI sites were chemically synthesized. The following oligonucleotides were used: Bottom strand, 5'-CGgttaacCGGG GTGAGGTAGTAGGTTGTGTGGTTTCAGGGCAGTGA TGTTGCCCCTCGGAAGATAACTATACAACCTACTGCC TTCCCTGctcgagCG-3'. Sequences corresponding to microRNA let-7 seed sequences are represented in capitalized and bold characters, and restriction enzyme sites are represented in lower case and bold characters. In order to produce the expression plasmid, the pairs of oligonucleotides were annealed as follows: Denaturation at $95^{\circ} \mathrm{C}$ for $5 \mathrm{~min}$; annealing at $58^{\circ} \mathrm{C}$ for $45 \mathrm{~min}$, then the oligonucleotides were inserted into the multiple cloning sites between the HpaI and XhoI sites in the pLL3.7 vector. The negative control plasmid, pLL3.7-miR-Mut, was constructed in a similar manner, with the exception that 22 nucleotides in sequences corresponding to microRNA let-7 seed sequences were mutated (TGAGGTAGTAGGTTGTGTGGTT was changed to TGTCCTAGAAGCATGAGAGCAT). The pLL3.7-miR-let-7 and pLL3.7-miR-Mut vectors were then recombined in the package cell lines, 293T, in order to create lentiviruses. Recombinant viruses were propagated in $293 \mathrm{~T}$ cells, and purified, and titered using standard methods, as described previously $(17,20)$. The corresponding viruses were termed Ldv-miR-let-7 or Ldv-miR-Mut. Cotransfection of EpSCs was conducted with $4 \times 10^{7} \mathrm{PFU} / \mathrm{ml}$ Ldv-miR-let -7 or Ldv-miR-Mut lentivirus, respectively, according to the manufacturer's instructions. Cells were seeded in a 6-well plate in 
DMEM (Sigma-Aldrich) supplemented with $10 \%$ fetal bovine serum (Hyclone Laboratories, Inc.), $100 \mathrm{U} / \mathrm{ml}$ penicillin and $100 \mu \mathrm{g} / \mathrm{ml}$ streptomycin (Hyclone Laboratories, Inc.), at $37^{\circ} \mathrm{C}$ in a humidified atmosphere of air with $5 \% \mathrm{CO}_{2}$, until they reached $80 \%$ confluence.

Reverse transcription-quantitative polymerase chain reaction (RT-qPCR) analysis. Total RNA from was isolated using TRIzol Reagent (Invitrogen Life Technologies, Carlsbad, CA, USA) according to the manufacturer's instructions. RNA samples were treated with Dnase I (Sigma-Aldrich) and reverse-transcribed into cDNA using the ReverTra Ace- $\alpha$ First Strand cDNA Synthesis kit (Toyobo, Co., Ltd., Osaka, Japan). RT-qPCR was conducted using a RealPlex4 real-time PCR detection system, obtained from Eppendorf Co., Ltd. (Hamburg, Germany), with SyBR Green RealTime PCR Master Mix (Toyobo, Co., Ltd.) used as the detection dye. RT-qPCR amplification was performed over 40 cycles with denaturation at $95^{\circ} \mathrm{C}$ for $15 \mathrm{sec}$ and annealing at $58^{\circ} \mathrm{C}$ for $45 \mathrm{sec}$. Target cDNA was quantified using the relative quantification method $(20,25)$. A comparative threshold cycle $(\mathrm{Ct})$ was used to determine gene expression relative to that of a control (calibrator), and steady-state mRNA levels were reported as an $\mathrm{n}$-fold difference relative to the calibrator. For each sample, the maker genes $\mathrm{Ct}$ values were normalized using the following formula: $\Delta \mathrm{Ct}=\mathrm{Ct}$ (gene) $-\mathrm{Ct}(18 \mathrm{sRNA})$. In order to determine relative expression levels, the following formula was used: $\Delta \Delta \mathrm{Ct}=\Delta \mathrm{Ct}(\mathrm{Ldv}-$ miR-let-7 group $)-\Delta \mathrm{Ct}(\mathrm{Ldv}-\mathrm{miR}-\mathrm{Mut}$ group). The values used to plot relative expressions of markers, were calculated using the expression $2^{-\Delta \Delta \mathrm{Ct}}$. The mRNA levels were calibrated based on levels of 18sRNA. The cDNA of each stem cell markers was amplified using the primers shown in Table I.

Western blotting analysis. Protein extracts were resolved using 12\% SDS-PAGE and transferred onto polyvinylidene difluoride (PVDF) membranes (Merck Millipore, Bedford, MA, USA). The PVDF membranes were blocked with WB blocking solution (Beyotime Biotechnology Co., Ltd, Zhejiang, China), and subsequently washed four times for $15 \mathrm{~min}$ with Tris-buffered saline with Tween-20 (TBST; Merck Millipore) at room temperature and incubated with the following primary antibodies: Rabbit anti-human p53 polyclonal antibody (cat. no. 12571), rabbit anti-human CCND1 polyclonal antibody (cat. no. 3300), rabbit anti-human cyclin-dependent kinase 4 (CDK4) polyclonal antibody (cat. no. 12790), rabbit anti-human E-cadherin polyclonal antibody (cat. no. 3195), rabbit anti-human claudin 1 (CLDN1) polyclonal antibody (cat. no. 4933), rabbit anti-human GAPDH polyclonal antibody (cat. no. 5174), all at 1:1,000 (Cell Signaling Technology, Inc., Danvers, MA USA). Following extensive washing, membranes were incubated with a peroxidase-conjugated goat anti-rabbit IgG secondary antibody (Santa Cruz Biotechnology, Inc., Santa Cruz, Ca, USA) for $1 \mathrm{~h}$. After washing four times for 15 min with TBST at room temperature, the immunoreactivity was visualized using an enhanced chemiluminescence (ECL kit, Pierce Biotechnology, Inc., Rockford, IL, USA).

Immunofluorescence staining analysis. The cultured cells were washed three times with PBS and fixed with $4 \%$
Table I. Primers used for RT-qPCR.

\begin{tabular}{ll}
\hline Gene & \multicolumn{1}{c}{ RT-qPCR primers $\left(5^{\prime} \rightarrow 3^{\prime}\right)$} \\
\hline$p 53$ & F: GCTTTCCACGACGGTGAC \\
& R: GCTCGACGCTAGGATCTGAC \\
FCND1 & R: TCCTCTCCAAAATGCCAGAG \\
& F: TGCAGTCCACATATGCAACA \\
CDK4 & R: GTCGGCTTCAGAGTTTCCAC \\
& F: CAGCCACCCGAGATTGAGCA \\
& R: TAGTAGCGACGGGCGGTGTG \\
Let-7b & F: TGAGGTAGTAGGTTGTGTGGTT \\
& R: GCTGTCAACGATACGCTACCTA
\end{tabular}

RT-qPCR, reverse transcription-quantitative polymerase chain reaction; $\mathrm{F}$, forward; $\mathrm{R}$, reverse; CCND1, cyclin D1; CDK4, cyclin-dependent kinase 4

paraformaldehyde (Sigma-Aldrich) for $30 \mathrm{~min}$. Following blocking, the cells were incubated overnight at $4{ }^{\circ} \mathrm{C}$ with primary antibodies as follows: Rabbit anti-human p53 polyclonal antibody (cat. no. 12571), rabbit anti-human CCND1 polyclonal antibody (cat. no. 3300), rabbit anti-human CDK4 polyclonal antibody (cat. no. 12790), rabbit anti-human E-cadherin polyclonal antibody (cat. no. 3195), rabbit anti-human CLDN1 polyclonal antibody (cat. no. 4933), rabbit anti-human GAPDH polyclonal antibody (cat. no. 5174), all at 1:1,000 (Cell Signaling Technology, Inc.) and then incubated with Cy3-conjugated goat anti-rabbit IgG secondary antibody (1:200; Abcam, Cambridge, UK) and $5 \mathrm{mg} / \mathrm{ml}$ DAPI (Sigma-Aldrich) at room temperature for $30 \mathrm{~min}$. The cells were then thoroughly washed with TBST and viewed through a fluorescence microscope (DMI3000; Leica, Allendale, NJ, USA).

Northern blotting. Northern blotting was conducted as previously described $(17,20)$. For all cell treatment groups, $20 \mu \mathrm{g}$ of total RNA was analyzed on a $7.5 \mathrm{M}$ urea, $12 \%$ PAA denaturing gel and transferred to a Hybond N+nylon membrane (Amersham, Freiburg, Germany). Membranes were cross-linked using ultraviolet light for $30 \mathrm{sec}$ at $1,200 \mathrm{~mJ} / \mathrm{cm}^{2}$ andhybridized tothe let $-7 b$ antisenseStarfireprobe, 5'-AACCACACAACCTACTACCTCA-3' (Sangon Biotech Co., Ltd, Shanghai, China) for the detection of 22-nucleotide let-7b fragments, according to the manufacturer's instructions. After washing, membranes were exposed to a Kodak XAR-5 film (Sigma-Aldrich) for 20-40 h. A human U6 snRNA probe (5'-GCAGGGGCCATGCTAATCTTCTCTGTATCG-3') was used as a positive control, with an exposure time of 15-30 min.

Flow cytometric (FCM) analysis of the cell cycle by PI staining. Each group of cells were seeded at $3 \times 10^{5}$ per well in 6-well plates and cultured until they reached $85 \%$ confluence. Each group of cells was washed three times with PBS, then collected by centrifugation (Allegra X-22R, Beckman Coulter, Brea, CA, USA) at $1,000 \mathrm{x} \mathrm{g}$ for $5 \mathrm{~min}$. The cell pellets were 

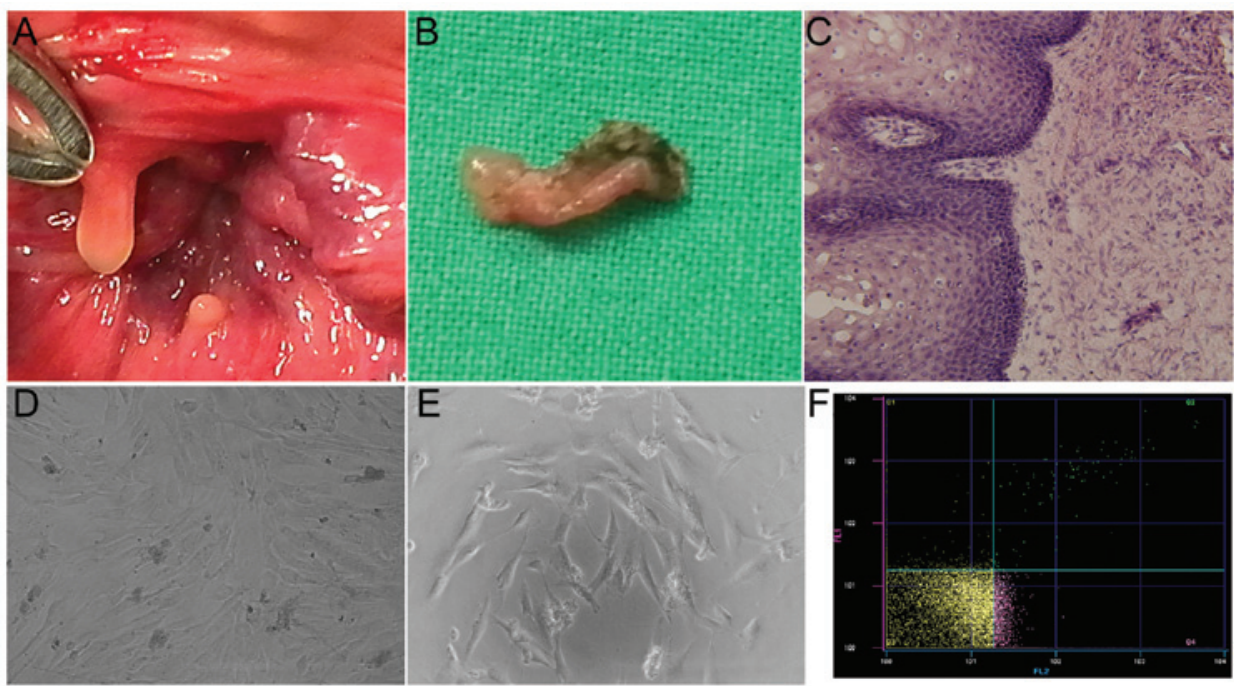

Figure 1. Characteristics of human hypertrophied anal papillae tissues and integrin $\alpha 6+/ \beta 1+$ EpSCs. (A) and (B) Human hypertrophied anal papillae tissue. (C) Histology of human hypertrophied anal papillae tissue. (D) Morphology of primary cells isolated and enriched from human hypertrophied anal papillae tissue. (E) Morphology of integrin $\alpha 6+/ \beta 1+E p S C s$ which were isolated and enriched from human hypertrophied anal papillae tissue. (F) Flow cytometry results, indicating that integrin $\alpha 6+/ \beta 1+E p S C$ represented $0.27 \pm 0.02 \%$ of total primary cells, which were isolated and enriched from human hypertrophied anal papillae tissue following sorting. EpSCs, epidermal stem cell markers.

the resuspended in $1 \mathrm{ml}$ of PBS, fixed in $70 \%$ ice-cold ethanol, and maintained in a freezer for $>48 \mathrm{~h}$. Prior to FCM analysis, the fixed cells were centrifuged, washed twice with PBS, and resuspended in PI staining solution (Sigma-Aldrich) containing $50 \mu \mathrm{l} / \mathrm{ml}$ PI and $250 \mu \mathrm{g} / \mathrm{ml}$ RNase A (Sigma-Aldrich). The cell suspension, which was maintained in darkness, was incubated for $30 \mathrm{~min}$ at $4^{\circ} \mathrm{C}$ and analyzed using the FACS (FACSAria, BD Biosciences, San Jose, CA, USA). A total of 20,000 events were acquired for analysis using CellQuest software version 2.1.0.

Statistical analysis. Each experiment was performed as least three times, and data are presented as the mean \pm standard error where applicable. Differences were evaluated using Student's t-test and $\mathrm{P}<0.05$ was considered to indicate a statistically significant difference. GraphPad Prism version 5.0 was also used for statistical analyses.

\section{Results}

$\alpha 6+/ \beta 1+$ EpSCs are present at low levels in hypertrophic anal papillae. The anal papillae examined in this study arose due to chronic inflammation caused by fibrous connective tissue hyperplasia. The anal papillae were cylindrical or walnut-shaped, with a large upper part and smaller lower part. The smooth surface was milky white, and no bleeding was observed (Fig. 1A and B). Pathological examination demonstrated significant endothelial hyperplasia in the anal papillae, accompanied by infiltration of inflammatory cells and vascular proliferation, although no cell heterogeneity was observed (Fig. 1C). A magnetic-activated cell sorting system was used to isolate and enrich the $\alpha 6+/ \beta 1+$ subpopulation from the hypertrophied anal papillae. Following isolation, cells were quantified using FCM. $\alpha 6+/ \beta 1+$ EpSCs represented $0.27 \pm 0.02 \%$ of the total population in five primary samples, whereas $\alpha 6$-/ $\beta 1$-cells represented $59.51 \pm 8.31 \%$ of
Table II. Assessment of EpSC marker expression using FCM.

\begin{tabular}{lcc}
\hline Biomarkers & $\alpha 6-/ \beta 1$-cells $(\mathrm{n}=5)$ & $\alpha 6+/ \beta 1+\operatorname{EpSCs}(\mathrm{n}=5)$ \\
\hline Integrin $\alpha 6$ & $1.155 \pm 0.015 \%$ & $60.645 \pm 4.955 \%$ \\
Integrin $\beta 1$ & $1.025 \pm 0.035 \%$ & $63.450 \pm 7.789 \%$ \\
$\beta$-caterin & $1.080 \pm 0.079 \%$ & $56.905 \pm 2.965 \%$ \\
\hline
\end{tabular}

EpSC, epidermal stem cell; FCM, flow cytometry.

the total population (Fig. 1D-F). These results demonstrated that $\alpha 6+/ \beta 1+$ epidermal stem cells (EpSCs), although occurring at a low frequency, may be successfully enriched using magnetic-activated cell sorting.

$\alpha 6+/ \beta 1+$ EpSCs express epidermal stem cell markers. FCM and immunofluorescent (IF) staining were used to compare the relative expression levels of epidermal stem cell markers in $\alpha 6+/ \beta 1+E p S C s$ and $\alpha 6-/ \beta 1$-cells. The IF assay confirmed that $\alpha 6+/ \beta 1+E p S C$ s expressed higher levels of the epidermal stem cell markers, integrin $\alpha 6$ and $\beta 1$ (Fig. 2A). FCM also demonstrated that the expression of integrin $\alpha 6, \beta 1$ and $\beta$-caterin was significantly higher in $\alpha 6+/ \beta 1+$ EpSCs than that in $\alpha 6$-/ $\beta 1$-cells (Fig. 2B; Table II). These results demonstrate that the $\alpha 6+/ \beta 1+$ subpopulation possesses epidermal stem cell characteristics.

$\alpha 6+/ \beta 1+E p S C$ s proliferate rapidly and express low levels of miRNA let-7. The proliferation rates of $\alpha 6+/ \beta 1+\mathrm{EpSCs}$ and $\alpha 6$-/ $\beta 1$-cells were examined for up to $72 \mathrm{~h}$ following passaging. Measurements were repeated in quintuplicate. No significant differences were observed in the total number of cells between the two groups at 0-12 h (Fig. 3A). However, between 24-72 h, $\alpha 6+/ \beta 1+$ EpSCs were found to divide significantly more rapidly than $\alpha 6-/ \beta 1$-cells (Table III). The miRNA RT-qPCR assay 
A

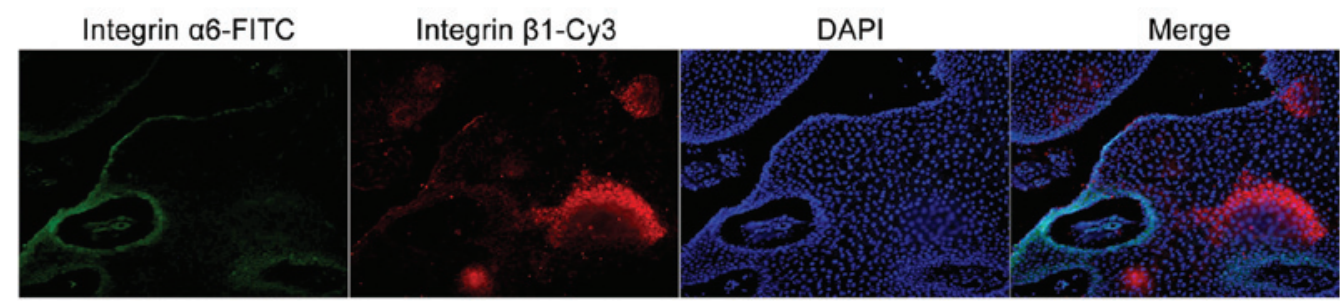

B
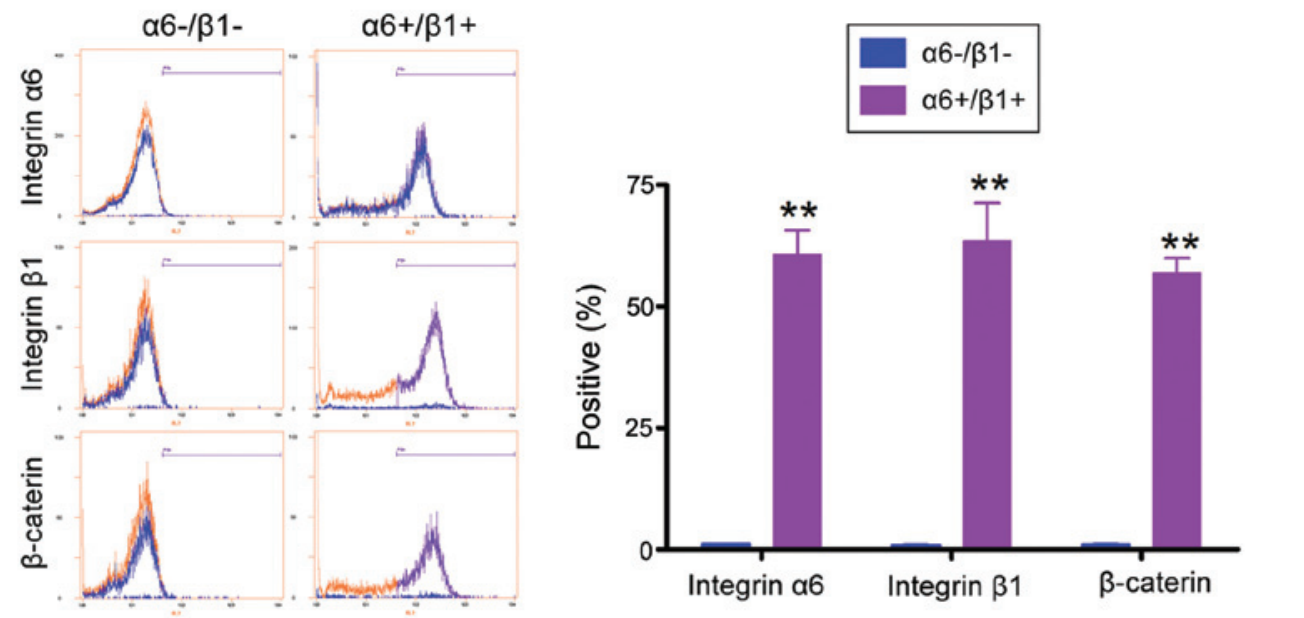

Figure 2. Analysis of EpSC expression of integrin $\alpha 6+/ \beta 1+$. (A) Immunofluorescent staining indicated that integrin $\alpha 6+/ \beta 1+$ EpSCs expressed high levels of integrin $\alpha 6$ and $\beta 1$. (B) FCM analysis of EpSC expression of integrin $\alpha 6+/ \beta 1+$. Expression of EpSC markers was higher in integrin $\alpha 6+/ \beta 1+E p S C s$ than that in integrin $\alpha 6$-/ק1-cells. EpSC, epidermal stem cell; FCM,flow cytometry; FITC, fluorescein isothiocyanate.

A

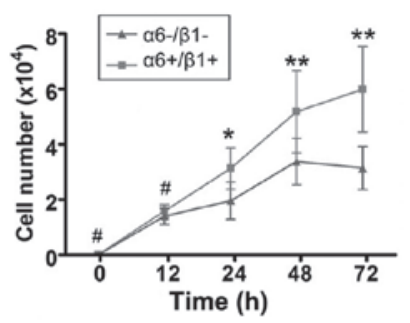

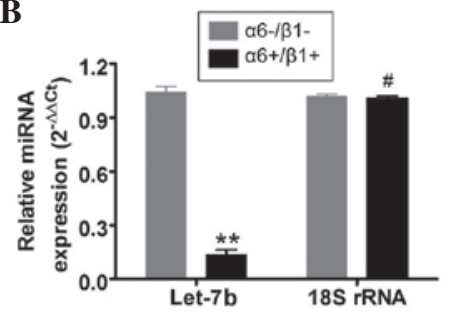

C

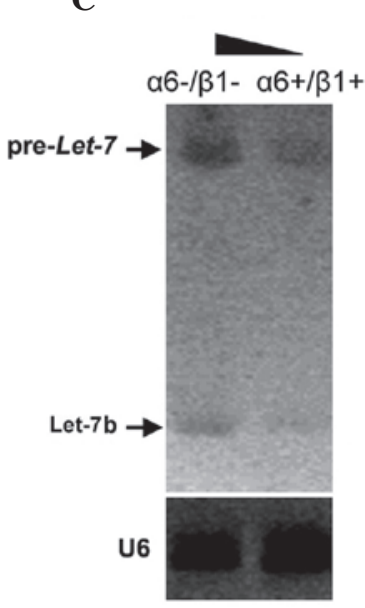

D
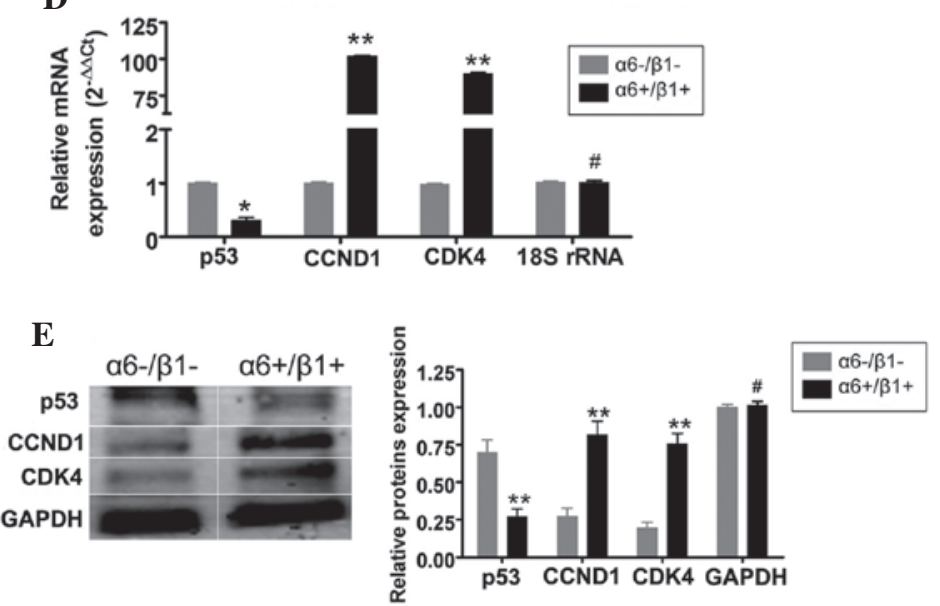

Figure 3. $\alpha 6+/ \beta 1+$ EpSCs proliferated more rapidly and expressed lower levels of microRNA let-7b than $\alpha 6-/ \beta 1-c e l l s$. (A) The proliferation rates of $\alpha 6+/ \beta 1+$ EpSCs and $\alpha 6$-/ $\beta 1$-cells were examined $12-72 \mathrm{~h}$ after passaging. Between $24-72 \mathrm{~h}, \alpha 6+/ \beta 1+E p S C$ s divided significantly more rapidly than $\alpha 6-/ \beta 1-$ cells. (B) An miRNA RT-qPCR assay demonstrated that expression of miRNA let-7b was markedly lower in $\alpha 6+/ \beta 1+$ EpSCs at $72 \mathrm{~h}$ compared with that in $\alpha 6-/ \beta 1$-cells. (C) Northern blot analysis revealed strong pre-miRNA let-7b and mature microRNA let- $7 b$ hybridization signals in $\alpha 6-/ \beta 1-c e l l s$ at $72 \mathrm{~h}$ compared with those $\alpha 6+/ \beta 1+$ EpSCs (D) RT-qPCR demonstrated that the mRNA expression of p53 was markedly lower in $\alpha 6+/ \beta 1+$ EpSCs at $72 \mathrm{~h}$ compared with that in $\alpha 6-/ \beta 1-$ cells. By contrast, mRNA expression of the cell cycle-related factors, CCND1 and CDK4, was significantly higher in $\alpha 6+/ \beta 1+$ EpSCs at $72 \mathrm{~h}$, compared with that in $\alpha 6-/ \beta 1-$ cells (E) Western blotting confirmed that p53 protein expression was significantly reduced in $\alpha 6+/ \beta 1+$ EpSCs. The expression of CCND1 and CDK4 proteins was significantly elevated in $\alpha 6+/ \beta 1+$ EpSCs. GAPDH served as a loading control ${ }^{* *} \mathrm{P}<0.01$, vs. $\alpha 6-/ \beta 1$-cells and ${ }^{*} \mathrm{P}<0.05$, vs. $\alpha 6-/ \beta 1-$ cells; $\mathrm{n}=3$ ). EpSCs, Epidermal stem cells; RT-qPCR, reverse transcription-quantitative polymerase chain reaction; CCND1, cyclin D1; CDK4, cyclin-dependent kinase 4. 
Table III. Cell proliferation assay.

Cell numbers $\left(\times 10^{4} / \mathrm{ml} ; \mathrm{n}=5\right)$

Time (hours) $\quad \alpha 6-/ \beta 1$-cells $\quad \alpha 6+/ \beta 1+$ EpSCs $\quad$ miR-Let-7b transcription group miR-Mut transcription group

\begin{tabular}{lcccc}
\hline 0 & 0 & 0 & 0 & 0 \\
12 & $1.39 \pm 0.29$ & $1.58 \pm 0.23$ & $1.29 \pm 0.27$ & $1.12 \pm 0.14$ \\
24 & $1.95 \pm 0.68$ & $3.12 \pm 0.75$ & $1.76 \pm 0.69$ & $2.02 \pm 0.91$ \\
48 & $3.38 \pm 0.84$ & $5.17 \pm 1.48$ & $3.19 \pm 0.63$ & $5.49 \pm 1.29$ \\
72 & $3.137 \pm 0.77$ & $5.98 \pm 1.55$ & $4.62 \pm 0.39$ & $6.54 \pm 1.32$ \\
\hline
\end{tabular}

EpSCs, epidermal stem cells; miR-Mut, Let-7b microRNA with a mutation in the Let-7b seed sequences.

A

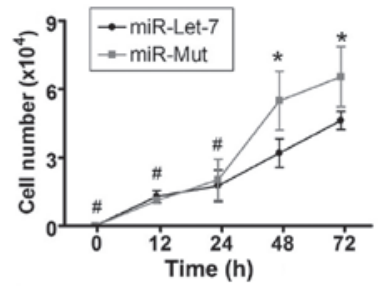

B

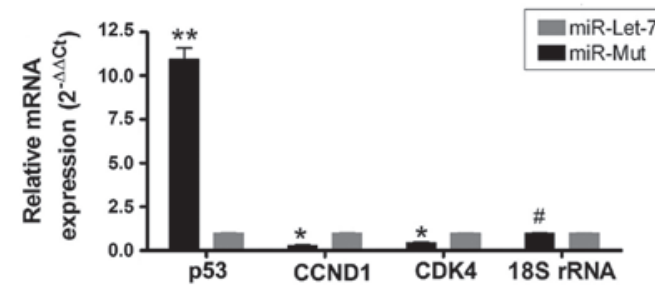

D

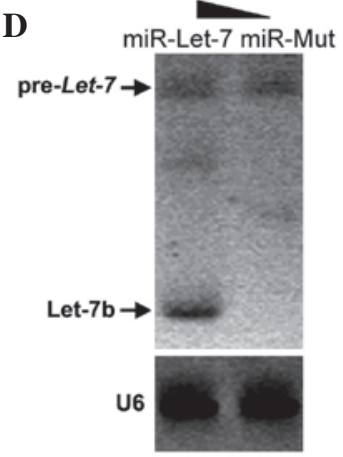

Figure 4. Overexpressed exogenous miRNA let-7b decreased proliferation of $\alpha 6+/ \beta 1+$ EpSCs by interfering with CCND1 expression. (A) Between $48-72$ h, $\alpha 6+/ \beta 1+$ EpSCs transfected with miR let-7b divided significantly more slowly than $\alpha 6+/ \beta 1+$ EpSCs transfected with miR-Mut $(*<<0.05$, vs. miR-Mut; $n=3$ ). (B) RT-qPCR demonstrated that p53 mRNA expression was markedly higher in $\alpha 6+/ \beta 1+$ EpSCs transfected with miR let-7b than that in $\alpha 6+/ \beta 1+E p S C s$ transfected with miR-Mut. By contrast, mRNA expression levels of the cell cycle-related factors, CCND1 and CDK4, were markedly lower in $\alpha 6+/ \beta 1+E p S C s$ transfected with miR let-7b than in $\alpha 6+/ \beta 1+$ EpSCs transfected with miR-Mut $\left({ }^{* *} \mathrm{P}<0.01\right.$, vs. miR-Mut and ${ }^{*} \mathrm{P}<0.05$, vs. miR-Mut; $\left.\mathrm{n}=3\right)$. (C) Western blotting confirmed that p53 protein expression was significantly increased in $\alpha 6+/ \beta 1+E p S C$ s transfected with miR let-7b. The expression of CCND1 and CDK 4 proteins was significantly decreased in $\alpha 6+/ \beta 1+$ EpSCs transfected with miR let $-7 b\left({ }^{* *} \mathrm{P}<0.01\right.$, vs. miR-Mut and ${ }^{*} \mathrm{P}<0.05$, vs. miR-Mut; $\left.\mathrm{n}=3\right)$. (D) Northern blotting demonstrated a strong let-7b hybridization signal in $\alpha 6+/ \beta 1+$ EpSCs transfected with miR let-7 compared with that in $\alpha 6+/ \beta 1+$ EpSCs transfected with miR-Mut. (E) FCM demonstrated that $\alpha 6+/ \beta 1+E p S C s$ transfected with miR let-7b were arrested in the G2/M phase, and that the percentage of cells in the S phase was significantly reduced. EpSCs, epidermal stem cells; CCND1, cyclin D1; CDK4, cyclin-dependent kinase 4; RT-qPCR, quantitative reverse transcription-polymerase chain reaction; FCM, flow cytometry.

showed that the expression of miRNA let-7b was markedly lower in $\alpha 6+/ \beta 1+$ EpSCs at $72 \mathrm{~h}$ than that in $\alpha 6-/ \beta 1-$ cells (Fig. 3B). Northern blot analysis demonstrated strong let-7b pre-miRNA and mature miRNA hybridization signals in $\alpha 6$-/ $/ \beta 1$-cells at $72 \mathrm{~h}$, compared with levels in $\alpha 6+/ \beta 1+$ EpSCs (Fig. 3C). RT-qPCR and western blotting were used to determine difference in the expression of cell cycle-related proteins in the different groups of cells. RT-qPCR showed that mRNA levels of $\mathrm{p} 53$, a protein involved in apoptosis, were markedly lower in $\alpha 6+/ \beta 1+\mathrm{EpSC}$ at $72 \mathrm{~h}$ compared with levels in $\alpha 6$-/ $\beta 1$-cells (Fig. 3D and E). By contrast, mRNA levels of the cell cycle-related proteins, CCND1 and CDK4, were markedly higher in $\alpha 6+/ \beta 1+E p S C s$ at $72 \mathrm{~h}$ than those in $\alpha 6-/ \beta 1$-cells (Fig. 3D and E). These findings were confirmed by western blotting (Fig. 3E). These results indicate that miRNA let- $7 b$ expression is reduced in $\alpha 6+/ \beta 1+\mathrm{EpSCs}$, stimulating the expression of cell cycle-related proteins and the proliferation of these cells.

Overexpression of exogenous miRNA let-7b decreases the proliferation rate of $\alpha 6+/ \beta 1+E p S C s$ by interfering with 
CCND1 expression. In order to determine whether the addition of exogenous miRNA let- $7 b$ influences $\alpha 6+/ \beta 1+\mathrm{EpSC}$ proliferation, recombinant lentiviruses expressing miR-let-7b or miR-Mut were transfected into $\alpha 6+/ \beta 1+$ EpSCs. Following expression of miRNA let-7b, no significant differences in the number of cells were detected between the two groups from 0-24 h (Fig. 4A). However, between 48-72 h, $\alpha 6+/ \beta 1+$ EpSCs transfected with miR-let-7b divided significantly less rapidly than $\alpha 6+/ \beta 1+$ EpSCs transfected with miR-Mut (Table III). The effects of the transfected miRNA on mRNA and protein expression were detected by RT-qPCR and northern blotting, and western blotting, respectively. The RT-qPCR results demonstrated that mRNA expression of p53 was markedly higher in $\alpha 6+/ \beta 1+$ EpSCs transfected with miR-let-7b than that in $\alpha 6+/ \beta 1+$ EpSCs transfected with miR-Mut (Fig. 4B). By contrast, mRNA expression of the cell cycle-related proteins, CCND1 and CDK4, was lower in $\alpha 6+/ \beta 1+$ EpSCs transfected with miR-let-7b than that in $\alpha 6+/ \beta 1+$ EpSCs transfected with miR-Mut (Fig. 4B). The western blotting results confirmed that p53 protein expression was significantly increased in $\alpha 6+/ \beta 1+$ EpSCs transfected with miR-let-7b compared with that in $\alpha 6+/ \beta 1+$ EpSCs transfected with miR-Mut (Fig. $4 \mathrm{C}$ ). The expression of the CCND1 and CDK4 proteins was significantly decreased in $\alpha 6+/ \beta 1+$ EpSCs transfected with miR-let- $7 b$ compared with that in $\alpha 6+/ \beta 1+$ EpSCs transfected with miR-Mut (Fig. 4E). Northern blotting demonstrated a strong let $-7 b$ hybridization signal in $\alpha 6+/ \beta 1+\mathrm{EpSCs}$ transfected with miR-let-7 compared with the signal in $\alpha 6+/ \beta 1+E p S C s$ transfected with miR-Mut (Fig. 4D). Furthermore, FCM demonstrated significant cell cycle arrest in $\alpha 6+/ \beta 1+E p S C$ s transfected with miR-let $-7 b$. Compared with $\alpha 6+/ \beta 1+$ EpSCs transfected with miR-Mut, $\alpha 6+/ \beta 1+$ EpSCs transfected with miR-let-7b were arrested in the $\mathrm{G} 2 / \mathrm{M}$ phase, and the percentage of S-phase cells in this group was significantly decreased (Fig. 4E). These results indicate that proliferation of the $\alpha 6+/ \beta 1+\mathrm{EpSC}$ subpopulation decreased when the expression of cell cycle-related proteins was suppressed by the addition of exogenous let- $7 b$ miRNA.

\section{Discussion}

Clinically, anal papillae may result in increases in local secretions, blood in the feces and anal itching (1-3). However, the pathogenesis of hypertrophic anal papillae remains unclear. The present study examined the mechanism underlying the development of hypertrophic anal papillae, with respect to the presence of EpSCs and miRNA-mediated epigenetics. In clinical practice, the growth of anal papilla in certain patients is rapid, and regrowth of new anal papilla tissue occurs in $<1$ year following surgical removal (1-3). It was hypothesized that the presence of cellular growth factors or stem cells, promoted cell proliferation and frequent regeneration. Due to the anatomical sites of anal papilla, it was hypothesized that EpSCs may be involved. There are various types of stem cells in the EpSC family, including follicular stem cells, hair follicle stem cells and sebaceous isthmus precursor stem cells $(5,6)$. EpSCs exhibit three basic stem cell characteristics, which are proliferation, self-renewal, as well as the ability to differentiate into keratinocytes $(5,6)$. In mammals, EpSCs produce a large number of mature skin cells in order to replace the epidermal and hair loss that occurs on a daily basis, and they exhibit a high metabolism. The skin is one of the most important regenerative organs in the body, and requires the rapid proliferation and differentiation potential of EpSCs. The present study found that $\alpha 6+/ \beta 1+$ EpSCs cells were present in patients with hypertrophic anal papillae. Following isolation and enrichment of these $\alpha 6+/ \beta 1+$ EpSCs, it was found that they exhibited a rapid rate of proliferation in vitro, and expressed elevated levels of cell cycle-related proteins. These results suggest that EpSCs are present in human hypertrophied anal papillae and that an increased rate of proliferation of these cells may be one of the causes of anal papilla hyperplasia.

There are a variety of mechanisms involved in the regulation of cell proliferation. Epigenetic regulation of transcription and of the expression of cell cycle-related genes is an area of current research. The miRNA, let-7b, is involved in the regulation of cell proliferation. The present study explored whether miRNA let-7b is involved in hypertrophic anal papilla hyperplasia. let-7b is a well-studied miRNA that is known to be involved in regulation of the cell cycle and development, and is underexpressed in various types of cancer (21). Restoration of normal let-7b expression has been shown to inhibit cancer growth by targeting various oncogenes and inhibiting the key regulators of a number of mitogenic pathways (21-24). The present study found that $\alpha 6+/ \beta 1+E p S C$ expression of endogenous microRNA let-7b was significantly lower than that in $\alpha 6-/ \beta 1$-cells. Furthermore, target genes of microRNA let-7b, including the cell cycle regulatory factors, CCND1 and CDK4, were upregulated in $\alpha 6+/ \beta 1+$ EpSCs compared with $\alpha 6-/ \beta 1$-cells. When the exogenous precursor microRNA let-7 was overexpressed in $\alpha 6+/ \beta 1+\mathrm{EpSCs}$, it was demonstrated that the proliferation of $\alpha 6+/ \beta 1+E p S C$ s was significantly less rapid than EpSCs transfected with miR-Mut. The expression of mature microRNA let-7b was significantly higher, while that of cyclin CCND1 and CDK4 was reduced. Compared with $\alpha 6+/ \beta 1+$ EpSCs transfected with miR-Mut, $\alpha 6+/ \beta 1+$ EpSCs transfected with miR-let-7b were arrested in the $\mathrm{G} 2 / \mathrm{M}$ phase, and the percentage of S-phase cells in this group was significantly reduced. These data indicate that proliferation of the $\alpha 6+/ \beta 1+$ EpSCs subpopulation was decreased when the level of endogenous cell cycle-related proteins was suppressed by overexpression of exogenous miRNA let-7b.

In conclusion, the present data suggest that two important mechanisms contribute to the development and recurrence of hypertrophic anal papillae. The first is hyperproliferation of EpSCs. The second is the expression of miRNA let-7b, which modulates expression of the cell cycle-related proteins, CCND1 and $\mathrm{CDK} 4$, and contributes to excessive cellular proliferation.

\section{Acknowledgements}

This study was supported by grants from the National Natural Science Foundation of China (grant nos. 81202811 and 81403401), the Project funded by China Postdoctoral Science Foundation (grant no. 2014M550250), Shanghai Municipal Health Bureau Fund (grant no. 20124320), Shanghai Municipal Health Bureau Fund (grant no. 20124Y013) and Shanghai 'Xing Lin Xin Xing' Fund (grant no. ZYSNXD011-RC-XLX $\mathrm{X}-20130025)$. 


\section{References}

1. Gupta PJ: Hypertrophied anal papillae and fibrous anal polyps, should they be removed during anal fissure surgery? World J Gastroenterol 10: 2412-2414, 2004.

2. Gupta PJ and Kalaskar S: Removal of hypertrophied anal papillae and fibrous anal polyps increases patient satisfaction after anal fissure surgery. Tech Coloproctol 7: 155-158, 2003.

3. Gupta PJ: A study of the symptomatology of hypertrophied anal papillae and fibrous anal polyps. Bratisl Lek Listy 106: 30-33, 2005.

4. Reiisi S, Esmaeili F and Shirazi A: Isolation, culture and identification of epidermal stem cells from newborn mouse skin. In vitro Cell Dev Biol Anim 46: 54-59, 2010.

5. Janes SM, Lowell S and Hutter C: Epidermal stem cells. J Pathol 197: 479-491, 2002.

6. Barthel R and Aberdam D: Epidermal stem cells. J Eur Acad Dermatol Venereol 19: 405-413, 2005.

7. Chen S, Takahara M, Kido M, et al: Increased expression of an epidermal stem cell marker, cytokeratin 19, in cutaneous squamous cell carcinoma. Br J Dermatol 159: 952-955, 2008

8. Luis NM, Morey L, Mejetta S, et al: Regulation of human epidermal stem cell proliferation and senescence requires polycomb-dependent and-independent functions of Cbx4. Cell Stem Cell 9: 233-246, 2011.

9. Watt FM: Role of integrins in regulating epidermal adhesion, growth and differentiation. EMBO J 21: 3919-3926, 2002.

10. Nanba D, Toki F, Matsushita N, Matsushita S, Higashiyama S and Barrandon Y: Actin filament dynamics impacts keratinocyte stem cell maintenance. EMBO Mol Med 5: 640-653, 2013.

11. Sumazin P, Yang X, Chiu HS, et al: An extensive microRNA-mediated network of RNA-RNA interactions regulates established oncogenic pathways in glioblastoma. Cell 147: 370-381, 2011

12. Poulton JS, Huang YC, Smith L, et al: The microRNA pathway regulates the temporal pattern of Notch signaling in Drosophila follicle cells. Development 138: 1737-1745, 2011.

13. Lei P, Li Y, Chen X, Yang S and Zhang J: Microarray based analysis of microRNA expression in rat cerebral cortex after traumatic brain injury. Brain Res 1284: 191-201, 2009.
14. Bartel DP: MicroRNAs: Genomics, biogenesis, mechanism, and function. Cell 116: 281-297, 2004.

15. Yoo AS, Sun AX, Li L, et al: MicroRNA-mediated conversion of human fibroblasts to neurons. Nature 476: 228-231, 2011.

16. Dai Y, Qiu Z, Diao Z, et al: MicroRNA-155 inhibits proliferation and migration of human extravillous trophoblast derived HTR-8/SVneo cells via down-regulating cyclin D1. Placenta 33: 824-829, 2012.

17. Liu T, Shen D, Xing S, et al: Attenuation of exogenous angiotensin II stress-induced damage and apoptosis in human vascular endothelial cells via microRNA-155 expression. Int J Mol Med 31: 188-196, 2012.

18. He L and Hannon GJ: MicroRNAs: Small RNAs with a big role in gene regulation. Nat Rev Genet 5: 522-531, 2004.

19. El Ouamari A, Baroukh N, Martens GA, Lebrun P, Pipeleers D and van Obberghen E: miR-375 targets 3'-phosphoinositide-dependent protein kinase-1 and regulates glucose-induced biological responses in pancreatic beta-cells. Diabetes 57: 2708-2717, 2008.

20. Liu T, Cheng W, Huang Y, Huang Q, Jiang L and Guo L: Human amniotic epithelial cell feeder layers maintain human iPS cell pluripotency via inhibited endogenous microRNA-145 and increased Sox 2 expression. Exp Cell Res 318: 424-434, 2012.

21. Barh D, Malhotra R, Ravi B and Sindhurani P: MicroRNA let-7: An emerging next-generation cancer therapeutic. Curr Oncol 17: 70-80, 2010.

22. Schultz J, Lorenz P, Gross G, Ibrahim S and Kunz M: MicroRNA let-7b targets important cell cycle molecules in malignant melanoma cells and interferes with anchorage-independent growth. Cell Res 18: 549-557, 2008.

23. Dangi-Garimella S, Yun J, Eves EM, et al: Raf kinase inhibitory protein suppresses a metastasis signalling cascade involving LIN28 and let-7. EMBO J 28: 347-358, 2009.

24. Yu F, Yao $\mathrm{H}$, Zhu $\mathrm{P}$, et al: Let-7 regulates self renewal and tumorigenicity of breast cancer cells. Cell 131: 1109-1123, 2007.

25. Liu T, Huang Y, Guo L, Cheng W and Zou G: CD44+/CD105+ human amniotic fluid mesenchymal stem cells survive and proliferate in the ovary long-term in a mouse model of chemotherapy-induced premature ovarian failure. Int J Med Sci 9: $592-602,2012$ 